\title{
A THEORY OF TYPE I SOLAR RADIO BURSTS
}

\author{
W. N.-C. SY \\ Division of Radiophysics, CSIRO, Sydney, Australia
}

\begin{abstract}
Proc. Astron. Soc. Australia). A theory is developed to account for the observed properties of type I storm bursts in terms of plasma radiation - that is, electromagnetic radiation at the electron plasma frequency resulting from the non-linear scattering of electron plasma waves on plasma ions. Now the average brightness temperature of a type I source is greater than $10^{9} \mathrm{~K}$, or even higher if, because of coronal scattering, the apparent source size is larger than the true source size. For brightness temperatures as high as $10^{9} \mathrm{~K}$ the non-linear scattering must be of the induced kind in which electromagnetic radiation below the frequency of the electron
\end{abstract}

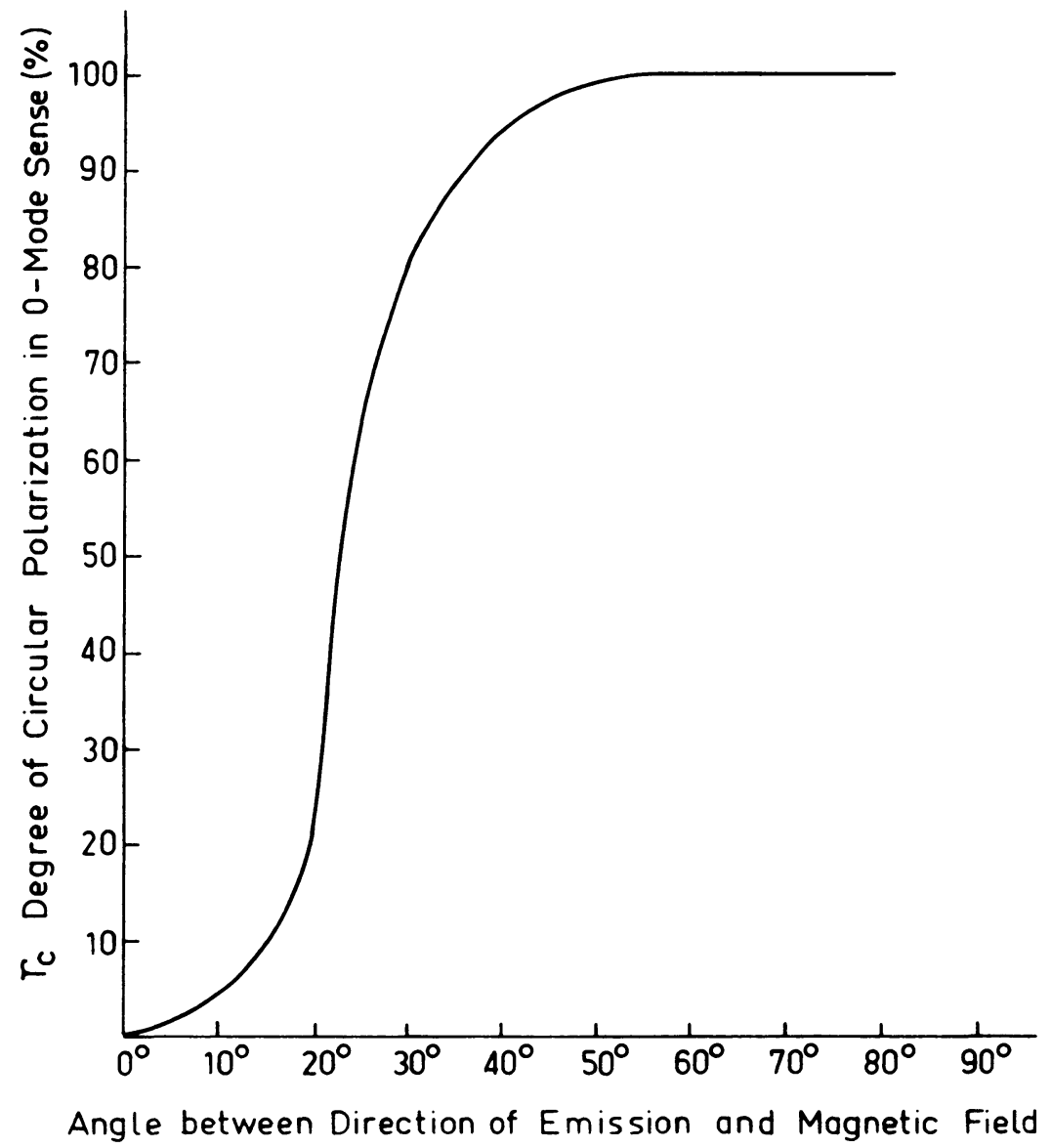

Fig. 1. The degree of circular polarization of amplified plasma radiation as a function of the angle between the directions of emission and of the magnetic field calculated for a simple model of a type I burst source. 
plasma waves is amplified. For such radiation to be strongly circularly polarized in the $o$-mode, as observed in type I bursts, requires that the amplification be more effective in the $o$-mode than in the $x$-mode (Figure 1). This is found to be so for plasma waves excited by electrons travelling parallel to the magnetic field. The electric field of the plasma waves is then also parallel to the magnetic field. The non-linear scattering is more efficient for that magnetoionic mode which has the greater component of electric field in the same direction. This mode is the $o$-mode.

\section{DISCUSSION}

Melrose: Your idea is interesting. How large does the optical depth have to be for the effect of the preferential amplification of the ordinary mode to be important?

$S y$ : The significant amplification of $o$-mode over $x$-mode depends on the angle of emission of the radiation with respect to the background magnetic field. At an emission angle of $45^{\circ}$ or greater the optical depth needs to be 4 or greater to give close to $100 \%$ polarization. 